TRANSACTIONS OF THE

AMERICAN MATHEMATICAL SOCIETY

Volume 352, Number 10, Pages 4451-4468

S 0002-9947(00)02634-9

Article electronically published on June 14, 2000

\title{
MANIFOLDS WITH MINIMAL RADIAL CURVATURE BOUNDED FROM BELOW AND BIG VOLUME
}

\author{
VALERY MARENICH
}

\begin{abstract}
We prove that a convergence in the Gromov-Hausdorff distance of manifolds with minimal radial curvature bounded from below by 1 to the standard sphere is equivalent to a volume convergence.
\end{abstract}

\section{INTRODUCTION}

A riemannian manifold $M^{n}$ has a minimal radial curvature $K_{o}^{\min }$ (with a base point $o$ ) bounded from below by $k, K_{o}^{\min } \geq k$ if for an arbitrary point $p$ and every minimal geodesic $\gamma(t), 0 \leq t \leq r$, connecting $o$ and $p$ the sectional curvature of $M^{n}$ is not less than $k$ in all two-dimensional directions which contain the vector $\dot{\gamma}(r)$. The class of manifolds with minimal radial curvature bounded from below is much bigger than the class of manifolds with sectional curvature bounded from below, but has many properties similar to this class or to the class of manifolds of Ricci curvature bounded from below. Some well-known results on geometry of riemannian manifolds of nonnegative sectional curvature, such as the Toponogov comparison theorem, estimates on diameter and radius, or the sphere theorem can be generalized to manifolds with non-negative minimal radial curvature; see M1, [M2], and [MS]. For instance, in our previous paper [MM] we obtained the following sphere theorem for such manifolds.

Theorem A. Let $M^{n}$ be a complete compact riemannian manifold without boundary of minimal radial curvature $K_{o}^{\min } \geq 1$ and $\operatorname{rad}\left(M^{n}\right) \geq \pi-\epsilon$. Then

1) for $\epsilon$ sufficiently small $M^{n}$ is homeomorphic to $S^{n}$ ( $M^{n}$ is a twisted sphere); and

2) for the Gromov-Hausdorff distance between $M^{n}$ and the standard sphere $S^{n}$ of constant curvature 1 we have

$$
d_{G H}\left(M^{n}, S^{n}\right) \leq C(\epsilon)
$$

for some function $C(\epsilon) \rightarrow 0$ as $\epsilon \rightarrow 0$.

This gives a generalization of corresponding results for manifolds of sectional curvature bounded from below; see $[\mathrm{GS}]$ and [GP]. For some other results see Corollaries B, C and D of the last theorem in [MM]. It is easy to see that according to Theorem A a Gromov-Hausdorff convergence of manifolds with $K_{o}^{\min } \geq 1$ to the standard sphere is equivalent to the convergence $\operatorname{rad}\left(M^{n}\right) \rightarrow \operatorname{rad}\left(S^{n}\right)$. In the

Received by the editors February 3, 1999.

1991 Mathematics Subject Classification. Primary 53C20, 53C21.

Key words and phrases. Sphere theorems, radial minimal curvature.

Supported by FAPERJ and CNPq. 
present paper we prove that the same convergence is equivalent to the convergence of volumes. This paper naturally continues [MM], so we preserve its notations and continue its numeration of results. Our main results are the following.

Theorem E. Let $M^{n}$ be a complete compact riemannian manifold without boundary of minimal radial curvature $K_{o}^{\text {min }} \geq 1$ and $d_{G H}\left(M^{n}, S^{n}\right)<\epsilon$. Then $\operatorname{vol}\left(S^{n}\right)-$ $C(\epsilon) \leq \operatorname{vol}\left(M^{n}\right) \leq \operatorname{vol}\left(S^{n}\right)$ for some function $C(\epsilon) \rightarrow 0$ as $\epsilon \rightarrow 0$.

Theorem F. Let $M^{n}$ be a complete compact riemannian manifold without boundary of minimal radial curvature $K_{o}^{\text {min }} \geq 1$ and $\operatorname{vol}\left(M^{n}\right) \geq \operatorname{vol}\left(S^{n}\right)-\epsilon$. Then $d_{G H}\left(M^{n}, S^{n}\right)<C(\epsilon)$ for some function $C(\epsilon) \rightarrow 0$ as $\epsilon \rightarrow 0$.

Again as in $\mathrm{MM}$, we would like to stress that above we do not require that the sectional curvatures of the manifolds under consideration are bounded from below. Due to Yamaguchi, with this extra condition Gromov-Hausdorff convergence $d_{G H}\left(M^{n}, S^{n}\right) \rightarrow 0$ in Theorem E by itself implies the Lipschitz convergence $d_{\text {Lip }}\left(M^{n}, S^{n}\right) \rightarrow 0$, see [Y2]; while Theorem F has the following corollary.

Corollary G. Let $M^{n}$ be a complete compact riemannian manifold without boundary of minimal radial curvature $K_{o}^{\text {min }} \geq 1, \operatorname{vol}\left(M^{n}\right) \geq \operatorname{vol}\left(S^{n}\right)-\epsilon$ and sectional curvature bounded from below, $\sec \left(M^{n}\right) \geq-k^{2}$. Then for $\epsilon$ sufficiently small $M^{n}$ is diffeomorphic to $S^{n}$, and for the Lipschitz distance between $M^{n}$ and the standard sphere $S^{n}$ we have $d_{\text {Lip }}\left(M^{n}, S^{n}\right)<C(\epsilon, k)$ for some function $C(\epsilon, k) \rightarrow 0$ as $\epsilon \rightarrow 0$.

Corollary G was previously obtained in [MS], together with the fact that $M^{n}$ is homeomorphic to $S^{n}$ if the volume of $M^{n}$ is bigger than $3 / 4$ of the volume of $S^{n}$. We obtain our Theorem $\mathrm{F}$ by only slightly improving at one point arguments from MS] in order to avoid the condition $\sec \left(M^{n}\right) \geq-k^{2}$ used there.

Theorem F and Corollary G generalize the well-known result by Otsu, Shiohama and Yamaguchi (see [OSY]) when $\sec \left(M^{n}\right) \geq 1$. It is also interesting to note that the equivalence of the Gromov-Hausdorff convergence and volume convergence which we prove is the same equivalence as under the lower Ricci curvature bound; i.e., changing in Theorems $\mathrm{E}$ and $\mathrm{F}$ our condition on the minimal radial curvature $K_{o}^{\min } \geq 1$ to the corresponding condition on the Ricci curvature $\operatorname{Ric}\left(M^{n}\right) \geq(n-$ 1 ), we arrive at the celebrated results by Colding [Cl1]- Cl3]. We have already mentioned in our previous paper [MM] this similarity of results for two classes of manifolds.

Note that, due to our Theorem A, a manifold $M^{n}$ is homeomorphic to $S^{n}$ if $M^{n}$ is sufficiently close to $S^{n}$ in the Gromov-Hausdorff distance. Thus, in proving both Theorems E and F we can assume that $M^{n}$ is homeomorphic to $S^{n}$.

Briefly the proof of Theorem E could be presented as follows. Take some $\epsilon<<$ $\sigma<<\xi$ playing a role of different geometric scales. First (see $\S \mathrm{I}$.4), we construct a $\xi$-partition of the sphere $S^{n}$ into small domains $\bar{B}^{\alpha}$, and measure their volumes by introducing in every $\bar{B}^{\alpha}$ distance coordinates $\bar{D}^{\alpha}: \bar{B}^{\alpha} \rightarrow R^{n}$ such that the maps $\bar{D}^{\alpha}$ are quasi-isometries on our sets $\bar{B}^{\alpha}$. Due to this construction the volume of $S^{n}$ almost equals the sum of volumes of $\bar{D}^{\alpha}\left(\bar{B}^{\alpha}\right)$. Second (see $\S$ I.3), we define for our manifold $M^{n}$ with $d_{G H}\left(M^{n}, S^{n}\right)<\epsilon$ a continuous map $V^{*}: S^{n} \rightarrow M^{n}$ almost inverse (on a $\sigma$-scale) to the partially defined quasi-isometry $V: M^{n} \rightarrow S^{n}$ from [MM] (see $\S I .2) 1]$ Then the family of sets $B^{\alpha}=V^{*}\left(\bar{B}^{\alpha}\right)$ provides an almost

\footnotetext{
${ }^{1}$ The construction of $V^{*}$ heavily relies on the "comparison angle almost non-increasing deformation" introduced in [MM], which we are slightly generalizing here.
} 
partition of $M^{n}$ such that the volume of $M^{n}$ almost equals the sum of volumes of $B^{\alpha}$. By construction the $B^{\alpha}$ are close in the Gromov-Hausdorff distance to the $\bar{B}^{\alpha}$, so that there exists a family of volume contracting maps $D^{\alpha}: B^{\alpha} \rightarrow R^{n}$ similar to the family of maps $\bar{D}^{\alpha}$ above and such that $\bar{D}^{\alpha}\left(\bar{B}^{\alpha}\right)$ and $D^{\alpha}\left(B^{\alpha}\right)$ are $\sigma$-close. Because the map $V^{*}$ is continuous $B^{\alpha}$ is homeomorphic to $\bar{B}^{\alpha}$, both are topological balls, and simple homotopy arguments show that the closeness in the Gromov-Hausdorff distance $d_{G H}\left(\bar{B}^{\alpha}, B^{\alpha}\right)<\sigma$ implies that the set $D^{\alpha}\left(B^{\alpha}\right)$ covers a substantial part of $\bar{D}^{\alpha}\left(\bar{B}^{\alpha}\right)$, yielding an inequality $\operatorname{vol}\left(B^{\alpha}\right)>(1-\Lambda(\epsilon)) \operatorname{vol}\left(\bar{B}^{\alpha}\right)$ for some $\Lambda(\epsilon) \rightarrow 0$ as $\epsilon \rightarrow 0$, which due to our choice $\epsilon<<\sigma<<\xi$ leads to the conclusion of Theorem E: $\operatorname{vol}\left(M^{n}\right)>\operatorname{vol}\left(S^{n}\right)-C(\epsilon)$ for some $C(\epsilon) \rightarrow 0$ as $\epsilon \rightarrow 0$.

It is my pleasure to thank the referee for suggestions and careful reading. I also would like to express my sincere gratitude to Sergio J. X. Mendonça for attracting my attention to the class of manifolds with minimal radial curvature bounded from below, and for much cooperation.

\section{Proof of Theorem E}

We begin with some notations and results established in previous papers.

1. Basic facts and notations. Comparison theorems. Denote by $\gamma_{p q}$ a geodesic in $M^{n}$ joining $p$ and $q$. For a triangle $\triangle_{p q r}$ in $M^{n}$ let $\triangle \bar{p} \bar{q} \bar{r}$ be a triangle in the sphere $S^{2}$ or $S^{n}$ of constant curvature 1 with the same lengths, called the comparison triangle of $\triangle_{p q r}$. By $\measuredangle q p r$ or simply by $\measuredangle p$ we denote the angle at $p$ of the triangle $\triangle_{p q r}$. The corresponding angle in $\triangle \bar{p} \bar{q} \bar{r}$, which we call the comparison angle, we denote by $\tilde{\measuredangle} q p r$ or simply by $\tilde{\measuredangle} p$. As was proved in [M2, for a manifold $M^{n}$ of minimal radial curvature $K_{o}^{\text {min }} \geq 1$ with a base point $o$ the Toponogov Comparison theorem is valid for triangles $\triangle_{o p q}$ in $M^{n}$ if one vertex is a base point $o$ and all sides are minimal geodesics. As before in [MM, we shall use this fact, referring to it as Proposition 1, see [MM].

Proposition 1. Let $M^{n}$ be a complete manifold with $K_{o}^{\text {min }} \geq c$, and let $\triangle_{o p q}$ be a triangle of minimal geodesic segments in $M^{n}$. Then there exists a comparison triangle $\triangle \bar{p} \bar{q} \bar{r}$ in the sphere $S^{2}$ of constant curvature 1 , and we have $\measuredangle o \geq \tilde{\measuredangle}$, $\measuredangle p \geq \tilde{\measuredangle} p$ and $\measuredangle q \geq \tilde{\measuredangle} q$.

(See Proposition 2 in [MM] for Alexandrov-Toponogov monotonicity of a comparison angle property, and Proposition 3 establishing the original Toponogov comparison theorem for manifolds of minimal radial curvature bounded from below.)

In a standard way, as simple consequences of Proposition 1 we have $\operatorname{diam}\left(M^{n}\right) \leq$ $\pi$ and $\operatorname{dist}(o, p)+\operatorname{dist}(o, q)+\operatorname{dist}(p, q) \leq 2 \pi$ for arbitrary points $o, p, q$ of $M^{n}$. In MS it is proved that the equality in any of these inequalities implies that $M^{n}$ is isometric to $S^{n}$ (see also our Corollary 1 in [MM]).

The radius $\operatorname{rad}\left(M^{n}\right)$ of the manifold $M^{n}$ is defined as $\inf _{x \in M} \sup _{y \in M} \operatorname{dist}(x, y)$, where by dist we denote the riemannian distance of $M^{n}$. In other words, $\operatorname{rad}\left(M^{n}\right)$ is the infimum of the values of the radius of balls that contain $M$. Again an easy application of Proposition 1 yields $\operatorname{rad}\left(M^{n}\right) \leq \pi$ for manifolds with $K_{o}^{\text {min }} \geq 1$, where $\operatorname{rad}\left(M^{n}\right)=\pi$ implies that $M^{n}$ is isometric to $S^{n}$.

In the same way it is easy to see that, for manifolds of minimal radial curvature $K_{o}^{\text {min }} \geq k$ the Bishop-Gromov volume comparison theorem is valid for balls with a center at the base point $o$, see [MS]. 
Proposition 4. Let $M^{n}$ be a manifold of minimal radial curvature $K_{o}^{\min } \geq k$ and $B(o, r)$ a metric ball in $M^{n}$ with center at the base point $o$ and radius $r$. Correspondingly, let $B(\bar{o}, r)$ be the $r$-ball in the manifold of constant curvature $k$. Then the function $\operatorname{vol}(B(o, r)) / \operatorname{vol}(B(\bar{o}, r))$ is monotone non-increasing.

To prove this statement it is sufficient to repeat the arguments of the proof of the Bishop-Gromov comparison theorem. Volume comparison and $\operatorname{diam}\left(M^{n}\right) \leq \pi$ lead to the inequalities

$$
\operatorname{vol}(B(o, r)) \leq \operatorname{vol}(B(\bar{o}, r))
$$

and

$$
\operatorname{vol}\left(M^{n}\right) \leq \operatorname{vol}\left(S^{n}\right)
$$

Gromov-Hausdorff distance. Recall that the Gromov-Hausdorff distance $d_{G H}\left(M^{n}, S^{n}\right)<\epsilon$ between $M^{n}$ and $S^{n}$ if there exist a metric space $Z$ and isometric embeddings $I_{M^{n}}: M^{n} \rightarrow Z$ and $I_{S^{n}}: S^{n} \rightarrow Z$ such that the Hausdorff distance $d_{H}$ between $I_{M^{n}}\left(M^{n}\right)$ and $I_{S^{n}}\left(S^{n}\right)$ in $Z$ is less than $\epsilon$, where for two subsets $A, B \subset Z$

$$
d_{H}(A, B)=\sup _{a \in A} \inf _{b \in B}\{\operatorname{dist}(a, b)\}+\inf _{a \in A} \sup _{b \in B}\{\operatorname{dist}(a, b)\} .
$$

Below we fix $Z, I_{M^{n}}: M^{n} \rightarrow Z, I_{S^{n}}: S^{n} \rightarrow Z$ and identify $I_{M^{n}}\left(M^{n}\right)$ with $M^{n}$, and $I_{S^{n}}\left(S^{n}\right)$ with $S^{n}$. We say that the set $A$ is close (or $d$-close) to the set $B$ if $d_{G H}(A, B)$ is small $\left(d_{G H}(A, B)<d\right)$.

Below we are assuming that $M^{n}$ is $\epsilon / 2$-close to $S^{n}$ and the base point $o$ is $\epsilon / 2$-close to $\bar{o}$. Then for an arbitrary point $x$ of $M^{n}$ there exists a point $\bar{x}$ of $S^{n}$ such that $\operatorname{dist}_{Z}(x, \bar{x})<\epsilon / 2$, and for the point $-\bar{x}$ antipodal to $\bar{x}$ in the sphere $S^{n}$, there exists some $x^{*}$ of $M^{n}$ with $\operatorname{dist}_{Z}\left(x^{*},-\bar{x}\right)<\epsilon / 2$. Due to the triangle inequality, for an arbitrary point $x$ of $M^{n}$ this provides us with a point $x^{*}$ such that $\operatorname{dist}\left(x, x^{*}\right)>\pi-\epsilon$, proving in particular that

$$
\operatorname{rad}\left(M^{n}\right)>\pi-\epsilon .
$$

$x^{*}$ always denotes the point (which need not be unique) such that $\operatorname{dist}\left(x, x^{*}\right)$ attains its maximum. Below by $\Lambda_{i}^{j}$ we denote the $j$ 's constant in $i$ 's lemma, and in all considerations below all geodesics are parameterized by an arc-length if it is not stated otherwise.

Again, as in [MM], we introduce together with $\epsilon$ three more parameters $\sigma, \xi$ and $\eta$ such that $\epsilon<<\sigma<<\xi<<\eta$ as $\epsilon \rightarrow 0$, and which will play together with $\epsilon$ the roles of different scales for our arguments. Among many possible choices we take $\epsilon=\sigma^{3}=\xi^{9}=\eta^{27}$ for definiteness. By $\sigma(x)$ we denote $\max \left\{\operatorname{dist}(o, x), \operatorname{dist}\left(o^{*}, x\right)\right\}$. It is usually assumed that $\sigma(x) \geq \sigma$.

2. Map $V: M^{n} \rightarrow S^{n}$. Inequality (1.3) allows us to use the following results and constructions from our previous paper [MM].

Lemma 1, $\mathrm{MM}$. If $M^{n}$ is a manifold of minimal radial curvature $K_{o}^{\text {min }} \geq 1$ and $\operatorname{rad}\left(M^{n}\right)>\pi-\epsilon$, then for an arbitrary point $x$ of $M$ and a point $x^{*}$ such that $\operatorname{dist}\left(x, x^{*}\right)>\pi-\epsilon$ the angle

$$
\tilde{\measuredangle}\left(x o x^{*}\right) \geq \pi-\Lambda_{1}(\epsilon), \quad \text { and } \quad \tilde{\measuredangle} o x o^{*} \geq \pi-\Lambda_{1}(\epsilon)
$$

for some function $\Lambda_{1}(\epsilon) \rightarrow 0$ as $\epsilon \rightarrow 0$, if $\sigma(x) \geq \sigma$. 
Lemma 2, $\mathrm{MM}$. Let $x$ and $y$ be two arbitrary points in $M^{n}$, and $\tilde{x}$ and $\tilde{y}$ two points in $S^{2}$ such that $\operatorname{dist}(\bar{o}, \tilde{x})=\operatorname{dist}(o, x), \operatorname{dist}(\bar{o}, \tilde{y})=\operatorname{dist}(o, y)$ and $\measuredangle(x o y)=$ $\measuredangle(\tilde{x} \bar{o} \tilde{y})$. If $\sigma(x), \sigma(y)>\sigma$, then $|\operatorname{dist}(\tilde{x}, \tilde{y})-\operatorname{dist}(x, y)|<\Lambda_{2}(\epsilon)$ for some function $\Lambda_{2}(\epsilon) \rightarrow 0$ as $\epsilon \rightarrow 0$.

Below we shall need also the following estimate.

Lemma 32. If $\triangle_{o x y}$ is some triangle in $M^{n}$ such that $\sigma<\operatorname{dist}(o, x)$, dist $(o, y)<$ $\pi-\sigma$ and $\triangle_{\bar{o} \bar{x} \bar{y}}$ its comparison triangle in $S^{n}$, then the angles of $\triangle_{\text {oxy }}$ are $\Lambda_{3}(\epsilon)$ close to the corresponding angles of $\triangle_{\bar{o} \bar{x} \bar{y}}$ for some function $\Lambda_{3}(\epsilon) \rightarrow 0$ having order $\sigma$ as $\epsilon \rightarrow 0$. For instance,

$$
0 \leq \angle(x \circ y)-\angle(\bar{x} \bar{o} \bar{y}) \leq \Lambda_{3}(\epsilon)
$$

for some $\Lambda_{3}(\epsilon) \rightarrow 0$ of order $\sigma$ as $\epsilon \rightarrow 0$.

Proof. Obviously, in proving the claim of the lemma we can assume that all comparison angles of the triangles $\triangle_{o x y}$ and $\triangle_{o y x^{*}}$ are bigger than $\sigma$ and less than $\pi-\sigma$. In a comparison sphere $S^{2}$ we construct a comparison triangle $\triangle_{\bar{o} \bar{x} \bar{y}}$ of the triangle $\triangle_{o x y}$ and denote $r=\operatorname{dist}(o, y), r_{1}=\operatorname{dist}\left(o, x^{*}\right)$ and $r_{2}=\operatorname{dist}(\bar{o},-\bar{x})$ for $x^{*}$ in $M^{n}$ such that $\operatorname{dist}\left(x, x^{*}\right)>\pi-\epsilon$. Let us construct also a comparison triangle $\triangle_{\bar{o} \bar{y} \bar{x}^{*}}$ in $S^{2}$ not overlapping $\triangle_{\bar{o} \bar{x} \bar{y}}$. Denote $d_{1}=\operatorname{dist}\left(y, x^{*}\right)$ and $d_{2}=\operatorname{dist}(\bar{y},-\bar{x})$. Then

$$
\left|r_{1}-r_{2}\right|<3 \epsilon
$$

and

$$
d_{1} \geq d_{2}-\epsilon
$$

Indeed,

$$
\begin{aligned}
r_{1}-r_{2} & =\operatorname{dist}\left(\bar{o}, \bar{x}^{*}\right)-\operatorname{dist}(\bar{o},-\bar{x})=\operatorname{dist}\left(\bar{o}, \bar{x}^{*}\right)-(\pi-\operatorname{dist}(\bar{o}, \bar{x})) \\
& =\left(\operatorname{dist}(o, x)+\operatorname{dist}\left(o, x^{*}\right)-\operatorname{dist}\left(x, x^{*}\right)\right)+\left(\operatorname{dist}\left(x, x^{*}\right)-\pi\right) .
\end{aligned}
$$

From $\pi-\epsilon<\operatorname{dist}\left(x, x^{*}\right)<\pi$ and the inequality $\operatorname{dist}(o, x)+\operatorname{dist}\left(o, x^{*}\right)+\operatorname{dist}\left(x, x^{*}\right) \leq$ $2 \pi$, from Proposition 1 we find that

$$
\operatorname{dist}(o, x)+\operatorname{dist}\left(o, x^{*}\right)-\operatorname{dist}\left(x, x^{*}\right) \leq 2 \epsilon,
$$

so that (1.6) gives (1.4), while (1.5) follows from

$$
\begin{aligned}
d_{1} & =\operatorname{dist}\left(\bar{y}, \bar{x}^{*}\right) \\
& =\left(\operatorname{dist}\left(\bar{x}, \bar{x}^{*}\right)-\operatorname{dist}(\bar{x}, \bar{y})\right)+\left(\operatorname{dist}(\bar{x}, \bar{y})+\operatorname{dist}\left(\bar{y}, \bar{x}^{*}\right)-\operatorname{dist}\left(\bar{x}, \bar{x}^{*}\right)\right) \\
& \geq \pi-\epsilon-\operatorname{dist}(\bar{x}, \bar{y})=\operatorname{dist}(\bar{y},-\bar{x})-\epsilon=d_{2}-\epsilon .
\end{aligned}
$$

Because $\tilde{\measuredangle}(x o y)=\measuredangle(\bar{x} \bar{o} \bar{y})=\pi-\measuredangle(\bar{y} \bar{o}(-\bar{x}))$, the claim of Lemma 3 will follow if only we prove that $\phi_{1}<\phi_{2}+\Lambda_{3}(\epsilon)$, where $\phi_{1}=\measuredangle\left(\bar{y} \bar{o} \bar{x}^{*}\right)$ and $\phi_{2}=\measuredangle(\bar{y} \bar{o}(-\bar{x}))$. Both triangles $\triangle_{\bar{y} \bar{o} \bar{x}^{*}}$ and $\triangle_{\bar{y} \bar{o}(-\bar{x})}$ have common side $\bar{o} \bar{y}$, according to (1.4) almost equal sides $\bar{o} \bar{x}^{*}$ and $\bar{o}(-\bar{x})$, while due to (1.5) the side $\bar{y} \bar{x}^{*}$ is almost bigger than the corresponding side $\bar{y}(-\bar{x})$. Now denote by $\tilde{x}$ the point of intersection of a geodesic $\gamma_{\bar{y}(-\bar{x})}$ with a geodesic $\gamma_{\bar{o} \bar{x}^{*}}$ or its continuation, i.e., $\tilde{x}=\gamma_{\bar{y}(-\bar{x})} \cap \gamma$, where by $\gamma$ we denote a geodesic connecting in $S^{2}$ two antipodal points $\bar{o}$ and $-\bar{o}$ and containing $\bar{x}^{*}$. First consider the case when $\tilde{x}$ belongs to the continuation of $\gamma_{\bar{o} \bar{x}^{*}}$. In this

\footnotetext{
${ }^{2}$ See also Lemma 12 of $[\mathrm{MM}]$.
} 
case the point $\bar{x}^{*}$ belongs to the interior of the triangle $\triangle_{\bar{o} \bar{y}(-\bar{x})}$, and because it is convex we have

$$
d_{1}+r_{1} \leq d_{2}+r_{2},
$$

which due to (1.4) and (1.5) immediately gives

$$
\left|d_{1}-d_{2}\right| \leq 3 \epsilon .
$$

Now find a point $\bar{z}$ on a geodesic $\gamma$ such that $\operatorname{dist}(\bar{o}, \bar{z})=\operatorname{dist}(\bar{o},-\bar{x})$. Due to (1.4) this point is close to $\bar{x}^{*}, \operatorname{dist}\left(\bar{x}^{*}, \bar{z}\right) \leq 3 \epsilon$. Hence, for $d_{3}=\operatorname{dist}(\bar{y}, \bar{z})$ we have

$$
\left|d_{2}-d_{3}\right| \leq 6 \epsilon \text {. }
$$

Consider two triangles, $\triangle_{\bar{y} \bar{o}-\bar{x}}$, and $\triangle_{\bar{y} \bar{o} \bar{z}}$. They have common side $\bar{y} \bar{o}$ and $\bar{o}-\bar{x}$ equals $\bar{o} \bar{z}$. Thus from the cosine formula we deduce that

$$
\cos \left(d_{2}\right)-\cos \left(d_{3}\right)=\sin (r) \sin \left(r_{2}\right)\left(\cos \left(\phi_{2}\right)-\cos \left(\phi_{1}\right)\right) .
$$

Applying (1.7) to the last formula, when $\epsilon, \sigma \rightarrow 0$, due to our conditions $\sigma<$ $r, r_{2}, \phi_{1}, \phi_{2}<\pi-\sigma$, we easily verify that $\left(\phi_{2}-\phi_{1}\right)$ is of order $\sigma$ as $\epsilon \rightarrow 0$, thus obtaining the claim of our lemma 3 In the case when the point $\tilde{x}$ belongs to the continuation of $\gamma_{\bar{o} \bar{x}^{*}}$ we apply the same arguments to the triangles $\triangle_{\bar{y}-\bar{o}-\bar{x}}$ and $\triangle_{\bar{y}-\bar{o} \bar{z}}$. Then the estimate

$$
\left|R_{1}-R_{2}\right| \leq 3 \epsilon
$$

for $R_{1}=\operatorname{dist}\left(-\bar{o}, \bar{x}^{*}\right)$ and $R_{2}=\operatorname{dist}(-\bar{o},-\bar{x})$, which follows from (1.4), yields the claim of the lemma. Lemma 3 is proved.

Remark 1. All constants $\Lambda_{i}(\epsilon), i=1,2,3$, above are at least of order $\sigma$ as $\epsilon \rightarrow 0$. We verified this for $\Lambda_{3}(\epsilon)$ in Lemma 3; for $i=1,2$ see [MM]. Below, see Lemma 9, we apply the estimates of Lemmas 1-3 to triangles $\triangle_{o x y}$ which are far from extremal, i.e., such that for at least one vertex $y$ of this triangle $\sigma(y)$ is close to $\pi / 2$ and the angle $\tilde{\measuredangle}($ xoy $)$ is close to $\pi / 2$. In this case the arguments of Lemmas $1-3$ (look at (1.8)) imply that the corresponding constants $\Lambda_{9}^{1}(\epsilon)$ and $\Lambda_{9}^{2}(\epsilon)$ are of order $\epsilon$.

In order to apply Lemmas $1-3$, we consider below, if it is not stated otherwise, only points from the set $M^{n}(\sigma)=M^{n} \backslash\left(B(o, \sigma) \cup B\left(o^{*}, \sigma\right)\right)$, i.e., satisfying the condition $\sigma(x)>\sigma$.

Map $V: M^{n}(\sigma) \rightarrow S^{n}$. From Lemma 1 it follows that the angle between any two minimal geodesics connecting $o$ and the point $x \in M^{n}(\sigma)$ is not bigger than $2 \Lambda_{1}(\epsilon)$ (for the proof, let $x^{*}$ be some point of $M^{n}$ such that $\operatorname{dist}\left(x, x^{*}\right)>\pi-\epsilon$, and let $\bar{Z}^{*}$ be a unit vector of direction of some minimal geodesic connecting $o$ and $x^{*}$. Then due to Lemma 1 all minimal geodesics from $o$ to $x$ have angles with $\bar{Z}^{*}$ bigger than $\pi-\Lambda_{1}(\epsilon)$, or every angle between two of them is not bigger than $2 \Lambda_{1}(\epsilon)$. Thus, using the same arguments as in the proof of the isotopy lemma 1.4 from [C], we see that for every constant $\Lambda$ there exists a continuous map sending the point $x$ to some unit vector $Z(x)$ such that the angle between $Z(x)$ and a direction of an arbitrary minimal geodesic from $o$ to $x$ is less than $2 \Lambda_{1}(\epsilon)+\Lambda$. Take $\Lambda=\Lambda_{1}(\epsilon)$, so that this angle is always less than $3 \Lambda_{1}(\epsilon)$. Set $\bar{V}(x)=\operatorname{dist}(o, x) Z(x)$. Then our map $V: M^{n}(\sigma) \rightarrow S^{n}$ is given by

$$
V(x)=\exp _{\bar{o}} \circ I \circ \bar{V}(x),
$$

\footnotetext{
${ }^{3}$ See similar estimates in Lemma 7 below.
} 
where $\bar{o}$ is some fixed point of $S^{n}$ and $I$ some fixed isometry between $T_{o} M^{n}$ and $T_{\bar{o}} S^{n}$.

For the map $V: M^{n}(\sigma) \rightarrow S^{n}$ the following was proved.

Lemma 4, $\mathrm{MM}$.

$$
|\operatorname{dist}(V(x), V(y))-\operatorname{dist}(x, y)|<\Lambda_{4}(\epsilon)
$$

for some function $\Lambda_{4}(\epsilon) \rightarrow 0$ as $\epsilon \rightarrow 0$.

Again $\Lambda_{4}(\epsilon)$ is of order $\sigma$ as $\epsilon \rightarrow 0$.

Comparison angle almost non-increasing deformation. As follows from our Theorem A (see Lemmas 8-11), for $\epsilon \rightarrow 0$ there exists some $\phi_{0}(\epsilon) \rightarrow 0$ (depending also on the dimension $n$ ), such that for an arbitrary vector $v$ of $T_{o} M^{n}$ there exist a point $p$ of $\Sigma=\left\{p \in M^{n} \mid \operatorname{dist}(p, o)=\pi / 2\right\}$ and a minimal geodesic $\gamma_{o p}$ with a direction at $o$ having an angle with $v$ less than $\phi_{0}(\epsilon)$. Take $z$ arbitrary, and find such $p$ for $v$ equal to the direction of some minimal geodesic $\gamma_{o z}$. Due to Proposition 1 , in the comparison triangle $\triangle_{\bar{o} \bar{p} \bar{z}}$ the angle $\angle(\bar{p} \bar{o} \bar{z})$ is not bigger than $\phi_{0}(\epsilon)$, and in the case $\operatorname{dist}(o, z) \leq \operatorname{dist}(z, p)$ this leads to the estimate

$$
\angle(p z o) \geq \angle(\bar{p} \bar{z} \bar{o}) \geq \pi-2 \phi_{0}(\epsilon) .
$$

For another arbitrary minimal geodesic $\gamma^{\prime}$ connecting $z$ and $o$ the comparison triangle of the triangle consisting of $\gamma_{o p}, \gamma_{z p}$ and $\gamma^{\prime}$ equals the previous one. Hence all minimal geodesics from the point $z$ to $o$ have an angle with the direction of $\gamma_{z p}$ bigger than $\pi-2 \phi_{0}(\epsilon)$, or the angle between the directions of two arbitrary minimal geodesics from $z$ to $o$ is not bigger than $4 \phi_{0}(\epsilon)$. For $z$ with $\sigma \leq \operatorname{dist}(o, z) \leq \pi-\sigma$ similar estimates follow from Lemma 1: all minimal geodesics from $z$ to $o$ have an angle with the direction of an arbitrary minimal geodesic connecting $z$ with $o^{*}$ which is bigger than $\pi-\Lambda_{1}(\epsilon)$. Taking $\Lambda_{5}(\epsilon)=\max \left\{4 \phi_{0}(\epsilon), 2 \Lambda_{1}(\epsilon)\right\}$, we conclude that for an arbitrary point $z$ with $\operatorname{dist}(o, z) \leq \pi-\sigma$ for directions $X_{1}$ and $X_{2}$ at the point $z$ of arbitrary minimal geodesics $\gamma_{1}$ and $\gamma_{2}$ connecting $z$ with $o$ the following is true:

$$
\measuredangle\left(X_{1}, X_{2}\right) \leq \Lambda_{5}(\epsilon)
$$

for some $\Lambda_{5}(\epsilon) \rightarrow 0$ as $\epsilon \rightarrow 0$. This allows us to construct the vector field $X$ on $M^{n} \backslash\left(\{o\} \cup B\left(o^{*}, \sigma\right)\right)$ in the following way. Take $i n j=\min \left\{\epsilon, r_{i n}\left(M^{n}\right)\right\}$ and for an arbitrary point $z$ in the $i n j$-neighborhood of $o$ let $-X(z)$ equal the direction of a unique minimal geodesic $\gamma_{z o}$. For a point $z$ from $M^{n} \backslash\left\{B(o, i n j) \cap B\left(o^{*}, \sigma\right)\right\}$ choose arbitrarily a vector $X(z)$ equal to the direction of some minimal geodesic $\gamma_{z o}$, and for arbitrary $\lambda<i n j$ define a vector field $X_{z, \lambda}$ depending on a parameter $\lambda$ in a $\lambda$-neighborhood $B(z, \lambda)$ of the point $z$ as follows: for $x \in B(z, \lambda)$ the vector $X_{z, \lambda}(x)$ at the point $x$ equals the parallel transport of $X(z)$ along the unique minimal geodesic from $z$ to $x$. Here the parameter $\lambda$ may depend on $z$. Because $M^{n} \backslash\left\{B(o, i n j) \cup B\left(o^{*}, \sigma\right)\right\}$ is compact, the covering $\cup B(z, \lambda(z))$ contains some finite subcovering $\bigcup B\left(z_{i}, \lambda\left(z_{i}\right)\right)$, and choosing some partition of unity $f_{i}: B\left(z_{i}, \lambda\right) \rightarrow R, f_{i}>0, \sum f_{i}(x) \equiv 1$, associated with this subcovering, we define the smooth vector field $X_{\lambda}(x)=\sum f_{i}(x) X_{z_{i}, \lambda\left(z_{i}\right)}$, where we denote $\lambda=\max \left\{\lambda\left(z_{i}\right)\right\}$.

Denote by $x(s)$ the integral trajectory of the constructed vector field $X_{\lambda}$. When $\operatorname{dist}(o, x(s)) \leq i n j$ this trajectory coincides with some unique minimal geodesic issuing from the point $o$. If $\operatorname{dist}(o, x(s)) \geq i n j$, applying the formula (6.5) of [MM] 
for the derivative of the distance function, we conclude from (1.10) that the distance function $\operatorname{dist}(o, x(s))$ is a strictly increasing function. Hence, every trajectory $x(s)$ is a union of two smooth curves belonging correspondingly to $B(o, i n j)$ and $M^{n} \backslash\left\{B(o, i n j) \cup B\left(o^{*}, \sigma\right)\right\}$, and such that the following is true.

Lemma 5. Let $x(s), 0 \leq s \leq s_{0}$, be the maximal integral trajectory of the vector field $X_{\lambda}$. The distance function dist $(o, x(s))$ is a strictly increasing function. For their left derivatives the following is true:

$$
\operatorname{dist}^{\prime}(o, x(s)) \geq \cos \left(\Lambda_{5}(\epsilon)\right)
$$

and $x\left(s_{0}\right)$ belongs to $\partial B\left(o^{*}, \sigma\right)$.

Proof. See the arguments from [MM] leading to Lemma 6 of $[\mathrm{MM}]$.

From the last lemma we see that the distance $\tau(s)=\operatorname{dist}(o, x(s))$ could be considered as a continuous (almost everywhere differentiable) parameter on $x(s)$ such that

$$
|\tau(s)-s| \leq \pi\left(1-\cos \left(\Lambda_{5}(\epsilon)\right)\right.
$$

It follows also that $s$ could be considered as an almost natural parameter on $x(s)$; for the length of $x(s)$ the following is true (see also Lemma 6 of [MM]).

Lemma 6. Let $x(s), 0 \leq s \leq s_{0}$, be the maximal integral trajectory of the vector field $X_{\lambda}$. Then for the length $L\left(s_{1}, s_{2}\right)$ of an arbitrary interval $x(s), s_{1} \leq s \leq s_{2}$, the following is true:

$$
\left|L\left(s_{1}, s_{2}\right)-\left(s_{2}-s_{1}\right)\right| \leq \Lambda_{6}(\epsilon)
$$

for some $\Lambda_{6}(\epsilon) \rightarrow 0$ as $\epsilon \rightarrow 0$.

Denote by $\phi_{\tau}: M^{n} \backslash B\left(o^{*}, \sigma\right) \rightarrow M^{n} \backslash B\left(o^{*}, \sigma\right)$ the shift on the parameter $\tau$ along integral trajectories $x(s)$, i.e., the map sending the point $x(s)$ to the point $x(s+\tau)$ when $s+\tau \leq s_{0}$, or to $x\left(s_{0}\right)$ when $s+\tau \geq s_{0}$. The most important property of this deformation is the following "comparison angle almost non-increasing". For a previous version see Lemma 7 of $\underline{\mathrm{MM}}$.

Lemma 7. Let $z(s)$ be the integral trajectory of the vector field $X_{\lambda}, x$ some fixed point, and $\phi(s)=\tilde{\measuredangle}(x o z(s))$ and $\psi(s)=\tilde{\measuredangle}(x z(s) o)$ the comparison angles $o$ and $z$ of the triangle $\triangle_{x o z(s)}$. Then:

1) If $\pi / 2 \leq \psi(s) \leq \pi-\sigma$, then $\phi(s)$ is an almost non-increasing function on $s$, i.e., for their left derivative the following is true:

$$
\phi_{-}^{\prime}(s) \leq \Lambda_{7}^{1}(\lambda)
$$

for some function $\Lambda_{7}^{1}(\lambda) \rightarrow 0$ as $\lambda \rightarrow 0$ and arbitrary $\sigma>0$.

2) If $\sigma \leq \psi(s) \leq \pi / 2$, then

$$
\phi_{-}^{\prime}(s) \leq \frac{1-\operatorname{dist}_{-}^{\prime}(o, z(s))}{\operatorname{dist}(o, z(s))} \operatorname{ctg}(\psi(s))+\Lambda_{7}^{2}(\lambda)
$$

for some $\Lambda_{7}^{2}(\lambda) \rightarrow 0$ as $\lambda \rightarrow 0$ and arbitrary $\sigma>0$.

Proof 4 . For $\operatorname{dist}(o, z(s)) \leq i n j$ the trajectory $z(s)$ coincides with a minimal geodesic issuing from a base point $o$, and the claim of the lemma coincides with a

\footnotetext{
${ }^{4}$ Here we go back to our original simple geometric arguments instead of the rather long and somewhat analytical proof which we choose in $\underline{\mathrm{MM}}$.
} 
monotonicity property of a comparison angle, see Proposition 2 in [MM]. Therefore, below we are assuming that $\operatorname{dist}(o, z(s))>i n j$. First we consider the case when the comparison angle $\psi=\tilde{\measuredangle}(x z o)$, i.e., the angle $\bar{z}$ of the comparison triangle $\triangle_{o x z}$, is not less than $\pi / 2$.

Denote $r(s)=\operatorname{dist}(o, z(s)), d(s)=\operatorname{dist}(x, z(s))$ and $z=z(s)$, and let $\triangle_{\bar{x} \bar{o} \bar{z}}$ be the comparison triangle in the sphere $S^{2}$. Let $\tilde{z}(s ")$ for $s " \nearrow s$ denote the point on a geodesic segment $\gamma_{\bar{o} \bar{z}}$ connecting $\bar{o}$ and $\bar{z}$ such that $\operatorname{dist}\left(\bar{o}, \tilde{z}\left(s^{\prime \prime}\right)\right)=\operatorname{dist}\left(o, z\left(s^{\prime \prime}\right)\right)$, i.e., $\operatorname{dist}\left(\tilde{z}\left(s^{\prime \prime}\right), \bar{z}\right)=\left(s-s^{\prime \prime}\right) r_{-}^{\prime}(s)+o(|s-s "|)$. The left derivative of the distance function $d(s)$ between $x$ and the point $z(s)$ of the integral trajectory of the vector field $X_{\lambda}$ equals

$$
d_{-}^{\prime}(s)=-\max \left\{\left(X_{\lambda}(z), \Gamma\right) \mid \Gamma \in O_{z x}\right\} ;
$$

see (6.5) in [MM], where $O_{z x}$ denotes the set of directions of all minimal geodesics from $z$ to $x$. By definition $X_{\lambda}(z)=f_{i}(z) X_{z_{i}, \lambda}(z)$, where $\sum_{i} f_{i}=1$ and $X_{z_{i}, \lambda}(z)$ are parallel transports of directions of some minimal geodesics connecting points $z_{i}$ with the point $o$, where $z_{i}$ tends to $z$ if $\lambda \rightarrow 0$. Because of this, as $\lambda \rightarrow 0$, directions $X_{z_{i}, \lambda}(z)$ tend to directions $X_{i}$ of some minimal geodesics from $z$ to $o$. Hence,

$$
d_{-}^{\prime}(s) \rightarrow-\max \left\{\left(f_{i} X_{i}, \Gamma\right) \mid \Gamma \in O_{z x}\right\} \quad \text { as } \quad \lambda \rightarrow 0 .
$$

Due to Proposition 1, the angle between arbitrary minimal geodesic connecting $z$ with $x$ and every direction $X_{i}$ (of some minimal geodesic from $z$ to $o$ ) is not less than the angle $\psi=\tilde{\measuredangle}(x z o)$, i.e., angle $\bar{z}$ of the comparison triangle $\triangle_{\bar{o} \bar{x} \bar{z}}$. Therefore, $\psi \geq \pi / 2$,

$$
\begin{aligned}
d\left(s^{\prime \prime}\right) & =d(s)+d_{-}^{\prime}(s)\left(s-s^{\prime \prime}\right)+o\left(\left|s-s^{\prime \prime}\right|\right) \\
& \geq d(s)-\left(s-s^{\prime \prime}\right) \cos \left(\psi+\omega_{1}(\lambda)\right)+o\left(\left|s-s^{\prime \prime}\right|\right) .
\end{aligned}
$$

for some $\omega_{1}(\lambda) \rightarrow 0$ as $\lambda \rightarrow 0$. By the same reasoning

$$
r_{-}^{\prime}(s) \rightarrow-\max \left\{\left(f_{i} X_{i}, \Gamma\right) \mid \Gamma \in O_{z o}\right\},
$$

or, because $\left|\left(f_{i} X_{i}, \Gamma\right)\right| \leq \sum_{i} f_{i}=1$ we have

$$
r\left(s^{\prime \prime}\right) \geq r(s)-\left(s-s^{\prime \prime}\right) .
$$

Now, construct in the sphere $S^{2}$ the comparison triangle $\triangle_{\bar{o} \bar{x} \bar{z}(s ")}$ to the triangle $\triangle_{o x z(s ")}$ and such that the point $\bar{z}\left(s^{\prime \prime}\right)$ belongs to the same hemisphere relative to the geodesic $\gamma_{\bar{o} \bar{x}}$ as $z(s)$, i.e., $\bar{z}\left(s^{\prime \prime}\right) \rightarrow \bar{z}(s)$ as $s^{\prime \prime} \nearrow s$. Then from (1.12)

$$
\operatorname{dist}\left(\bar{x}, \bar{z}\left(s^{\prime \prime}\right)\right) \geq d(s)-\left(s-s^{\prime \prime}\right) \cos \left(\psi+\omega_{1}(\lambda)\right)+o\left(\left|s-s^{\prime \prime}\right|\right),
$$

and from (1.13)

$$
\operatorname{dist}\left(\bar{z}(s), \bar{z}\left(s^{\prime \prime}\right)\right) \leq\left(s-s^{\prime \prime}\right)+o\left(\left|s-s^{\prime \prime}\right|\right) .
$$

By definition the angle $\measuredangle(\bar{x} \bar{z} \tilde{z})=\psi$, and because for the point $\tilde{z}$ from (1.13) we have

$$
\operatorname{dist}\left(\bar{z}(s), \tilde{z}\left(s^{\prime \prime}\right)\right) \leq\left(s-s^{\prime \prime}\right)+o\left(\left|s-s^{\prime \prime}\right|\right) .
$$

we conclude that

$$
\begin{aligned}
\operatorname{dist}\left(\bar{x}, \tilde{z}\left(s^{\prime \prime}\right)\right) & =d(s)-\operatorname{dist}\left(\bar{z}(s), \tilde{z}\left(s^{\prime \prime}\right)\right) \cos (\psi)+o\left(\left|s-s^{\prime \prime}\right|\right) \\
& \leq d(s)-\left(s-s^{\prime \prime}\right) \cos (\psi)+o\left(\left|s-s^{\prime \prime}\right|\right)
\end{aligned}
$$


$\psi \geq \pi / 2$. Inequalities (1.14), (1.17) show that the point $\bar{z}\left(s^{\prime \prime}\right)$ having the same distance to $\bar{o}$ as the point $\tilde{z}(s ")$ at the same time has distance to $\bar{x}$ almost bigger than the point $\tilde{z}\left(s^{\prime \prime}\right)$ which belongs to the geodesic interval $\gamma_{\bar{o} \bar{z}}$,

$$
\begin{aligned}
& \operatorname{dist}\left(\bar{x}, \bar{z}\left(s^{\prime \prime}\right)\right)-\operatorname{dist}\left(\bar{x}, \tilde{z}\left(s^{\prime \prime}\right)\right) \\
& \quad \geq\left(s-s^{\prime \prime}\right)\left(\cos \left(\psi+\omega_{1}(\lambda)\right)-\cos (\psi)\right)+o\left(\left|s-s^{\prime \prime}\right|\right) \\
& \quad \geq-\left(s-s^{\prime \prime}\right) \omega_{1}(\lambda)+o(|s-s "|)
\end{aligned}
$$

for some function $\omega_{1}(\lambda) \rightarrow 0$ as $\lambda \rightarrow 0$. Therefore, the point $\bar{z}\left(s^{\prime \prime}\right)$ belongs to the exterior of the comparison triangle $\triangle_{\bar{o} \bar{x} \bar{z}}$, or belongs to the interior of this triangle, but has a distance less than $\left(s-s^{\prime \prime}\right)(\sin (\psi))^{-1} \omega_{1}(\lambda)$ to the side $\gamma_{\bar{o} \bar{z}}$. Indeed, consider in $S^{2}$ a circle of points having distance $r\left(s^{\prime \prime}\right)$ to the point $\bar{o}$. Both $\bar{z}\left(s^{\prime \prime}\right)$ and $\tilde{z}\left(s^{\prime \prime}\right)$ belong to this circle, and the angle between $\gamma_{\bar{x} \tilde{z}(s ")}$ and this circle tends to $\psi-\pi / 2$ as $s$ " $\nearrow s$. Thus the first variation formula gives

$$
\left|\operatorname{dist}\left(\bar{x}, \bar{z}\left(s^{\prime \prime}\right)\right)-\operatorname{dist}\left(\bar{x}, \tilde{z}\left(s^{\prime \prime}\right)\right)\right|=\operatorname{dist}\left(\bar{z}\left(s^{\prime \prime}\right), \tilde{z}\left(s^{\prime \prime}\right)\right) \sin (\psi)+o\left(\operatorname{dist}\left(\bar{z}\left(s^{\prime \prime}\right), \tilde{z}\left(s^{\prime \prime}\right)\right)\right) \text {. }
$$

Because $\operatorname{dist}(\bar{o}, \bar{z}) \geq i n j$, this easily implies that the angle $\measuredangle\left(\bar{x} \bar{o} \bar{z}\left(s^{\prime \prime}\right)\right)$ is almost bigger than $\measuredangle\left(\bar{x} \bar{o} \tilde{z}\left(s^{\prime \prime}\right)\right)=\measuredangle(\bar{x} \bar{o} \bar{z}(s))$, i.e., for some constant $\Lambda$

$$
\measuredangle(\bar{x} \bar{o} \tilde{z}(s)) \leq \measuredangle\left(\bar{x} \bar{o} \bar{z}\left(s^{\prime \prime}\right)\right)+\Lambda \frac{\left(s-s^{\prime \prime}\right)}{\operatorname{inj} \sin (\psi)} \omega_{1}(\lambda) .
$$

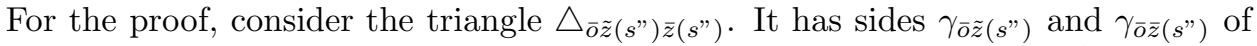
order $i n j$, while the third side $\gamma_{\tilde{z}\left(s^{\prime \prime}\right) \bar{z}\left(s^{\prime \prime}\right)}$ is of order $\left(s-s^{\prime \prime}\right)(\sin (\psi))^{-1} \omega_{1}(\lambda) \rightarrow 0$ as $\lambda \rightarrow 0$. Applying sphere geometry to this triangle, from $\psi \leq \pi-\sigma$ we arrive at (1.20), which obviously leads to the claim of the lemma when $\pi / 2 \leq \psi \leq \pi-\sigma$ :

$$
\phi_{-}^{\prime}(s) \leq \Lambda_{7}^{1}(\lambda),
$$

where we put $\Lambda_{7}^{1}(\lambda)=\Lambda(i n j)^{-1}(\sin (\psi))^{-1} \omega_{1}(\lambda) \rightarrow 0$ as $\lambda \rightarrow 0$ and for arbitrary $\sigma>0$.

When $\sigma \leq \psi \leq \pi / 2$, then instead of (1.12) we have

$$
d\left(s^{\prime \prime}\right) \geq d(s)-\left(s-s^{\prime \prime}\right) \cos \left(\psi+\omega_{2}(\lambda)\right)+o\left(\left|s-s^{\prime \prime}\right|\right)
$$

for some $\omega_{2}(\lambda) \rightarrow 0$ as $\lambda \rightarrow 0$, and accordingly

$$
\operatorname{dist}\left(\bar{x}, \bar{z}\left(s^{\prime \prime}\right)\right) \leq d(s)-\left(s-s^{\prime \prime}\right) \cos \left(\psi+\omega_{2}(\lambda)\right)+o\left(\left|s-s^{\prime \prime}\right|\right) .
$$

Instead of (1.13) we use

$$
\operatorname{dist}\left(z(s), z\left(s^{\prime \prime}\right)\right) \geq r_{-}^{\prime}(s)\left(s-s^{\prime \prime}\right)+o\left(\left|s-s^{\prime \prime}\right|\right) .
$$

Then $\measuredangle(\bar{x} \bar{z} \tilde{z})=\psi$ yields in the case $\psi \leq \pi / 2$ the following inequality:

$$
\begin{aligned}
\operatorname{dist}\left(\bar{x}, \tilde{z}\left(s^{\prime \prime}\right)\right) & =d(s)-\operatorname{dist}\left(\bar{z}(s), \tilde{z}\left(s^{\prime \prime}\right)\right) \cos (\psi)+o\left(\left|s-s^{\prime \prime}\right|\right) \\
& \leq d(s)-r_{-}^{\prime}(s)\left(s-s^{\prime \prime}\right) \cos (\psi)+o\left(\left|s-s^{\prime \prime}\right|\right) .
\end{aligned}
$$

Now inequalities (1.21) and (1.23) in the same way as above show that the point $\bar{z}(s ")$ having the same distance to $\bar{o}$ as the point $\tilde{z}(s ")$ at the same time has distance to $\bar{x}$ almost bigger than the point $\tilde{z}\left(s^{\prime \prime}\right)$ which belongs to the geodesic interval $\gamma_{\bar{o} \bar{z}}$,

$$
\begin{array}{r}
\operatorname{dist}\left(\bar{x}, \bar{z}\left(s^{\prime \prime}\right)\right)-\operatorname{dist}\left(\bar{x}, \tilde{z}\left(s^{\prime \prime}\right)\right) \geq\left|1-r_{-}^{\prime}(s)\right|\left(s-s^{\prime \prime}\right) \cos (\psi) \\
+\left(s-s^{\prime \prime}\right)\left(\cos \left(\psi+\omega_{2}(\lambda)\right)-\cos (\psi)\right)+o\left(\left|s-s^{\prime \prime}\right|\right) .
\end{array}
$$




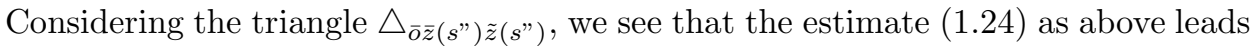
to the following estimate on its side $\gamma_{\bar{z}\left(s^{\prime \prime}\right) \tilde{z}\left(s^{\prime \prime}\right)}$ :

$$
\left.\operatorname{dist}\left(\bar{z}\left(s^{\prime \prime}\right), \tilde{z}\left(s^{\prime \prime}\right)\right) \leq\left(s-s^{\prime \prime}\right) \frac{1-r_{-}^{\prime}(s)}{r(s)} \operatorname{ctg}(\psi)+\left(s-s^{\prime \prime}\right) \Lambda_{7}^{2}(\lambda)\right)+o\left(s-s^{\prime \prime}\right),
$$

which in turn implies the following estimate on the derivative of the comparison angle: $\phi(s)=\measuredangle(\bar{x} \bar{o} \tilde{z}(s))$

$$
\phi_{-}^{\prime}(s) \leq \frac{1-r_{-}^{\prime}(s)}{r(s)} \operatorname{ctg}(\psi)+\Lambda_{7}^{2}(\lambda)
$$

for some $\Lambda_{7}^{2}(\lambda) \rightarrow 0$ as $\lambda \rightarrow 0$ for arbitrary $\sigma>0$. Lemma 7 is proved.

Lemma 8. For an arbitrary trajectory $z(s)$ and a point $x$ such that $\operatorname{dist}(o, z(s)) \leq$ $\operatorname{dist}(o, x)-\sin (\phi) \operatorname{dist}(o, x)$, where $\phi<\pi / 2$, the comparison angle $\phi(s)=\tilde{\measuredangle}(x o z(s))$ is an almost non-increasing function:

$$
\phi_{-}^{\prime}(s) \leq \Lambda_{7}^{1}(\lambda),
$$

for some function $\Lambda_{7}^{1}(\lambda) \rightarrow 0$ as $\lambda$.

Proof. For the proof, compare the triangle $\triangle_{o x z(s)}$ with a plane triangle $\triangle_{o^{\prime} x^{\prime} z^{\prime}(s)}$ with the same sides. The conditions $\operatorname{dist}(o, z(s)) \leq \operatorname{dist}(o, x)-\sin (\phi) \operatorname{dist}(o, x)$ and $\phi<\pi / 2$ lead to $\measuredangle\left(o^{\prime}\right)<\pi / 2$ and $\psi^{\prime}=\measuredangle\left(z^{\prime}(s)\right)>\pi / 2$. Applying Proposition 1, we have $\psi(s) \geq \psi^{\prime}>\pi / 2$, and the claim follows from Lemma 7 .

3. Almost isometry $V^{*}: S^{n} \rightarrow M^{n}$. Let $\bar{o}$ be some point of $S^{n}$ which is $\epsilon / 2$-close to the base point $o$ of $M^{n}$. Take some $n$-cross $\left\{\bar{P}_{i}, i=1, \ldots, n\right\}$ in the equatorial sphere $S^{n-1}$ of $S^{n}$, considering $\bar{o}$ as a pole, i.e., the set of points such that

$$
\operatorname{dist}\left(\bar{o}, \bar{P}_{i}\right) \equiv \pi / 2, \quad \operatorname{dist}\left(\bar{P}_{i}, \bar{P}_{j}\right) \equiv \pi / 2 \quad \text { for } \quad i \neq j,
$$

and find points $P_{i}$ in $M^{n}$ which are $\epsilon / 2$-close to them. Due to Lemmas 2 and 3 we see that the angles $\measuredangle\left(P_{i} o P_{j}\right)$ are almost equal to $\measuredangle\left(\bar{P}_{i} \bar{o} \bar{P}_{j}\right)$, i.e., for some constant $\Lambda_{9}^{1}(\epsilon)$ of order $\epsilon$, see Remark 1 above, we have

$$
\left|\measuredangle\left(P_{i} o P_{j}\right)-\measuredangle\left(\bar{P}_{i} \bar{o} \bar{P}_{j}\right)\right| \leq \Lambda_{9}^{1}(\epsilon) .
$$

Therefore, their exists an isometry $I: T_{\bar{o}} S^{n} \rightarrow T_{o} M^{n}$ such that for unit directions $\bar{e}_{i}$ of minimal geodesics $\gamma_{\bar{o} \bar{P}_{i}}$ in $S^{n}$ connecting points $\bar{o}$ and $\bar{P}_{i}$ and unit vectors $e_{i}$ of directions of arbitrary minimal geodesics $\gamma_{o P_{i}}$ in $M^{n}$ connecting points $o$ and $P_{i}$ the following is true:

$$
\measuredangle\left(e_{i}, I\left(\bar{e}_{i}\right)\right) \leq \Lambda_{9}^{2}(\epsilon),
$$

for some $\Lambda_{9}^{2}(\epsilon) \rightarrow 0$ of order $\epsilon$ as $\epsilon \rightarrow 0$. In particular, $e_{i}$ is a base of a tangent plane $T_{o} M^{n}$ for $\epsilon$ small enough. Now, let $\tilde{x}$ be some point of $S^{n}$ and $x$ an arbitrary point of $M^{n}$ which is $\epsilon / 2$-close to it and such that $\operatorname{dist}(\bar{o}, \tilde{x}), \operatorname{dist}(-\bar{o}, \tilde{x})$ and $\operatorname{dist}(o, x), \operatorname{dist}\left(o^{*}, x\right)$ are bigger than $\sigma$.

From Lemma 3 we conclude that for an arbitrary minimal geodesic $\gamma_{o x}$ between $o$ and $x$, for an arbitrary $i$, we have

$$
\left|\measuredangle\left(\dot{\gamma}_{o x}, e_{i}\right)-\measuredangle\left(\dot{\gamma}_{\bar{o} \tilde{x}}, \bar{e}_{i}\right)\right| \leq \Lambda_{9}^{3}(\epsilon),
$$

for some $\Lambda_{9}^{3}(\epsilon) \rightarrow 0$ of order $\sigma$ as $\epsilon \rightarrow 0$. Because every unit vector of $T_{o} M^{n}$ is determined by angles with the base vectors $e_{i}$, we are able to prove the following statement. 
Lemma 9. Let $\tilde{x}$ be some point of $S^{n}$ and $x$ an arbitrary point of $M^{n}$ which is $\epsilon / 2$ close to it and such that $\operatorname{dist}(\bar{o}, \tilde{x}), \operatorname{dist}(-\bar{o}, \tilde{x})$ and $\operatorname{dist}(o, x), \operatorname{dist}\left(o^{*}, x\right)$ are bigger than $\sigma$. Then, for an arbitrary minimal geodesic $\gamma_{o x}$ between $o$ and $x$,

$$
\measuredangle\left(\dot{\gamma}_{o x}, I\left(\dot{\gamma}_{\bar{o} \tilde{x}}\right) \leq \Lambda_{9}(\epsilon)\right.
$$

for some $\Lambda_{9}(\epsilon)$ of order $\sigma$ as $\epsilon \rightarrow 0$.

Now we define the map $V^{*}: S^{n} \rightarrow M^{n}$. Without loss of generality we may assume that $\Lambda_{9}(\epsilon) \geq 2 \sigma$. According to Lemma 7 there exists $\lambda$ in the definition of the field $X_{\lambda}$ so small that

$$
\pi \Lambda_{7}^{1}(\lambda)<\sigma<\Lambda_{9}(\epsilon) .
$$

Definition 1. For a point $\tilde{x}=\exp _{\bar{o}}(d \bar{v})$ of $S^{n}$, where $\bar{v}$ is a unit vector, we denote by $V^{*}(\tilde{x})$ the point

$$
V^{*}(\tilde{x})=\phi_{s(d)}\left(\exp _{o}(i n j I(\bar{v}))\right) \quad \text { for } \quad s(d)=d-i n j-4 \pi \Lambda_{9}(\epsilon) .
$$

Or $V^{*}(\tilde{x})=\phi_{s(d)}\left(x^{*}\right)$, where $d$ equals the distance between $\bar{o}$ and $\tilde{x}$, and $x^{*}$ is the point of $M^{n}$ very near to the base point $o$ and such that the unique minimal geodesic from $o$ to $x^{*}$ has direction $I(\bar{v})$, where $\bar{v}$ is the direction of a minimal geodesic from $\bar{o}$ to $\tilde{x}$.

Remark 2. We cannot control the behavior of an arbitrary geodesic $\gamma$ issuing from $o$ if $\gamma$ is not minimal, e.g., for such $\gamma$ the "comparison angle almost non-increasing" property proved in Lemma 7 above could be wrong. This is the reason why we are using our deformation $\phi_{s}$ instead of an exponential map.

Lemma 10. For all points $\tilde{x}$ from $S^{n}$ with $\xi<\operatorname{dist}(\bar{o}, \tilde{x})<\pi-\xi$ we have

$$
\operatorname{dist}\left(\tilde{x}, V^{*}(\tilde{x})\right)<\Lambda_{10}(\epsilon)
$$

for some $\Lambda_{10}(\epsilon)$ of order $\sigma$ as $\epsilon \rightarrow 0$.

Proof. For a point $\tilde{x}$ find some point $x$ of $M^{n}$ which is $\epsilon / 2$ close to it. Due to the last lemma,

$$
\measuredangle\left(\gamma_{o x}, I\left(\dot{\gamma}_{\bar{o} \tilde{x}}\right)<\Lambda_{9}(\epsilon),\right.
$$

or for the point $\left.x^{*}=\exp _{o}\left(\operatorname{inj} I\left(\dot{\gamma}_{\bar{o} \tilde{x}}\right)\right)\right)$ belonging to a geodesic issuing from $o$ with a direction $I(\bar{v})$, where $\bar{v}$ is the direction of $\gamma_{\bar{o} \tilde{x}}$, we have

$$
\measuredangle\left(\gamma_{o x^{*}}, \dot{\gamma}_{o x}\right)<\Lambda_{9}(\epsilon) \text {. }
$$

Take the trajectory $x^{*}(s), i n j \leq s \leq s(d)$, of the vector field $X_{\lambda}$ issuing from the point $x^{*}=x^{*}(i n j)$, and consider the comparison angle $\phi(s)=\tilde{\measuredangle}\left(x o x^{*}(s)\right)$. Because of the last estimate,

$$
\operatorname{dist}(o, x) \sin \left(\measuredangle\left(\gamma_{o x^{*}}, \dot{\gamma}_{o x}\right)\right)<\pi \Lambda_{9}(\epsilon)<\xi-\epsilon<\operatorname{dist}(o, x)-\operatorname{dist}\left(o, x^{*}(i n j)\right)
$$

for $\epsilon$ sufficiently small, because $\Lambda_{9}(\epsilon)$ is of order $\sigma$. Thus, due to Lemma 8 , at the initial moment $\phi^{\prime}(i n j) \leq \Lambda_{7}^{1}(\epsilon)$. Now, if $\left[i n j, s_{1}\right]$ denotes the maximal interval such that

$$
\phi(s) \leq 3 \Lambda_{9}(\epsilon) \quad \text { for all } \quad 0 \leq s \leq s_{1},
$$

we obtain from our definition (1.26)

$$
\operatorname{dist}(o, x) \sin \left(\measuredangle\left(\gamma_{o x^{*}(s)}, \dot{\gamma}_{o x}\right)\right)<3 \pi \Lambda_{9}(\epsilon)<(d-s(d)),
$$


implying that for all $i n j \leq s \leq s_{1}$ the conditions of Lemma 8 are satisfied. Hence, applying Lemmas 7-8 we conclude that for all $i n j \leq s \leq s_{1}$

$$
\phi(s) \leq \phi(i n j)+\pi \Lambda_{7}^{1}<2 \Lambda_{9}(\epsilon),
$$

or that $s_{1}$ is not a maximal value unless $s_{1}=s(d)$. Hence (1.27) is true for all $0 \leq s \leq s(d)$. Therefore,

$$
\measuredangle\left(\dot{\gamma}_{o x^{*}(s(d))}, \dot{\gamma}_{o x}\right)<3 \Lambda_{9}(\epsilon)
$$

or

$$
\measuredangle\left(\dot{\gamma}_{o V^{*}(\tilde{x})}, \dot{\gamma}_{o x}\right)<3 \Lambda_{9}(\epsilon)
$$

where $\Lambda_{9}(\epsilon)$ is of order $\sigma$ when $\epsilon \rightarrow 0$. Consider the triangle $\triangle_{o x V^{*}(\tilde{x})}$. From Lemma 6 we see that it is almost equilateral:

$$
\left|\operatorname{dist}(o, x)-\operatorname{dist}\left(o, V^{*}(\tilde{x})\right)\right| \leq \Lambda_{6}(\epsilon)+(d-s(d)),
$$

and due to (1.28) it has small angle at the vertex $o$. Thus using Proposition 1 we conclude that its side $\gamma_{x V^{*}(\tilde{x})}$ is small:

$$
\operatorname{dist}\left(x, V^{*}(\tilde{x})\right)<\Lambda_{10}(\epsilon)
$$

where $\Lambda_{10}(\epsilon)$ is of the same order $\sigma$ as $\epsilon \rightarrow 0$. Lemma 10 is proved.

4. Nearly cube partition of the sphere. Fix some point $\bar{o}$ of the sphere $S^{n}$, and denote by $\left\{\phi_{1}, \ldots, \phi_{n}\right\}$, where $0 \leq \phi_{1} \leq 2 \pi$ and $0 \leq \phi_{i} \leq \pi, i=2, \ldots, n$, a system of polar "coordinates" on $S^{n}$ such that $\bar{o}$ has zero coordinates. If Sing denotes the set of points of the sphere $S^{n}$ where some of these coordinates equal zero, then $S^{n} \backslash$ Sing is a cell, and the map sending the point $p$ to its coordinates $\left\{\phi_{1}(p), \ldots, \phi_{n}(p)\right\}$ provides a diffeomorphism $\Phi:\left(S^{n} \backslash\right.$ Sing $) \rightarrow \Pi$ between $S^{n} \backslash$ Sing and an open cube $\Pi=(0,2 \pi) \times(o, \pi) \times(0, \pi) \times \ldots \times(0, \pi)$. Denote by $\Pi_{\xi}=$ $(\xi, 2 \pi-\xi) \times(\xi, \pi-\xi) \times(\xi, \pi-\xi) \times \ldots \times(\xi, \pi-\xi)$ the set of all interior points of $\Pi$ having distance greater than $\xi$ to the boundary of $\Pi$. In what follows $\xi \rightarrow 0$, and without loss of generality we may assume below that the number $\pi / \xi$ is an integer. For $\xi$-partitions $\xi=\phi_{i}^{1}<\phi_{i}^{2}<\ldots<\phi_{i}^{N}$ of the intervals $(\xi, \pi-\xi)$ for $i>1$ and $(\xi, 2 \pi-\xi)$ for $i=1$ correspondingly, we denote by

$$
\Pi\left(k_{1}, k_{2}, \ldots, k_{n}\right)=\left(\phi_{1}^{k_{1}}, \phi_{1}^{k_{1}}+\xi\right) \times\left(\phi_{2}^{k_{2}}, \phi_{2}^{k_{2}}+\xi\right) \times \ldots \times\left(\phi_{n}^{k_{n}}, \phi_{n}^{k_{n}}+\xi\right)
$$

the cubes of the corresponding partition of $\Pi_{\xi}$, which are products of the partitions of the intervals described above. Denote by $\alpha=\left\{k_{1}, \ldots, k_{n}\right\}$ the multi-index of the cube of this partition and by $\Pi^{\alpha}$ the corresponding cube. Using the coordinate map $\Phi:\left(S^{n} \backslash\right.$ Sing $) \rightarrow \Pi$, we define $S_{\xi}^{n}=\Phi^{-1}\left(\Pi_{\xi}\right)$ and its partition $\bar{B}^{\alpha}=\Phi^{-1}\left(\Pi^{\alpha}\right)$. By $\bar{B}_{\xi}^{\alpha}$ we denote the subset of $\bar{B}^{\alpha}$ consisting of all interior points with a distance at least $\xi$ to a boundary $\partial \bar{B}^{\alpha}$ of $\bar{B}^{\alpha}$. In every set $\bar{B}_{\xi}^{\alpha}$ we choose some point $\bar{z}_{\alpha}$ and the orthonormal base $e_{i}^{\alpha}, i=1, \ldots, n$, at this point. Let $\bar{p}_{i}^{\alpha}=\exp _{\bar{z}^{\alpha}}\left(\eta e_{i}^{\alpha}\right)$ and define by $\bar{d}_{i}^{\alpha}(\bar{z})=\operatorname{dist}\left(\bar{z}, \bar{p}_{i}^{\alpha}\right)$ the distance from $\bar{z}$ to the point $\bar{p}_{i}^{\alpha}$. By definition $\bar{B}^{\alpha}$ and $\left\{\bar{p}_{i}^{\alpha}, i=1, \ldots, n\right\}$ belong to a $\xi+\eta$-neighborhood of $\bar{z}^{\alpha}$, and for $\xi+\eta<<\pi / 2$ all functions $\bar{d}_{i}^{\alpha}$ are smooth on $\bar{B}^{\alpha}$; for an arbitrary $\bar{z} \in \bar{B}^{\alpha}$ there exists for every $i$ a unique minimal geodesic $\bar{\gamma}_{\bar{z}, i}$ connecting $\bar{z}$ with $\bar{p}_{i}^{\alpha}$, whose direction $\bar{X}_{i}(\bar{z})$ at the point $\bar{z}$ is the gradient of $\bar{d}_{i}^{\alpha}$. Moreover, because $\bar{B}^{\alpha}$ belongs to a $\xi$-neighborhood of $\bar{z}^{\alpha}$, in all triangles $\triangle_{\bar{z} \bar{z}^{\alpha} \bar{p}_{i}^{\alpha}}$ the side $\bar{z} \bar{z}^{\alpha}$ has length less than $\xi$, which is small compared to the length $\eta$ of $\bar{z}^{\alpha} \bar{p}_{i}^{\alpha}$ due to our choice of $\eta=\xi^{1 / 3}$. Therefore, all triangles $\triangle_{\bar{z} \bar{z}^{\alpha} \bar{p}_{i}^{\alpha}}$ are very thin, which easily leads to the following statement. 
Lemma 11. For an arbitrary $\bar{z}$ of $\bar{B}^{\alpha}$ the unitary vectors $\bar{X}_{i}(\bar{z}), i=1, \ldots, n$, form an almost orthogonal base, i.e.,

$$
\left|\left(\bar{X}_{i}(\bar{z}), \bar{X}_{j}(\bar{z})\right)-\delta_{i j}\right| \leq \xi_{1}(\epsilon)
$$

for some function $\xi_{1}(\epsilon) \rightarrow 0$ as $\epsilon \rightarrow 0$ and not depending on $\alpha$.

For a point $\bar{z}$ of $\bar{B}^{\alpha}$, denote by $\bar{D}^{\alpha}$ the map sending $\bar{z}$ to $\left\{\bar{d}_{1}^{\alpha}(\bar{z}), \ldots, \bar{d}_{n}^{\alpha}(\bar{z})\right\}$. As a corollary of the last lemma we conclude that this map is an almost isometry and, in particular, deduce that the volumes of $\bar{B}^{\alpha}$ and $\bar{D}^{\alpha}\left(\bar{B}^{\alpha}\right)$ are almost equal. We sum up all volume inequalities in the following lemma.

Lemma 12. For some function $\xi_{2}(\epsilon) \rightarrow 0$ as $\epsilon \rightarrow 0$

1) $1-\xi_{2}(\epsilon) \leq\left|\operatorname{vol}\left(\bar{B}^{\alpha}\right) / \operatorname{vol}\left(\bar{D}^{\alpha}\left(\bar{B}^{\alpha}\right)\right)\right| \leq 1+\xi_{2}(\epsilon)$,

2) $\left|\operatorname{vol}\left(S^{n}\right)-\sum_{\alpha} \operatorname{vol}\left(\bar{D}^{\alpha}\left(\bar{B}^{\alpha}\right)\right)\right| \leq \xi_{2}(\epsilon)$, and

3) $\operatorname{vol}\left(\bar{B}_{\mu}^{\alpha}\right) \geq\left(1-\xi_{2}(\mu)\right) \operatorname{vol}\left(\bar{B}^{\alpha}\right)$, where by $\bar{B}_{\mu}^{\alpha}$ we denote the subset of $\bar{B}^{\alpha}$ of all interior points having distance bigger than $\mu$ to the boundary of $\bar{B}^{\alpha}$.

Remark 3. Clearly, the same construction of a partition $B^{\alpha}$ and maps $D^{\alpha}: B^{\alpha} \rightarrow$ $R^{n}$ similar to $\bar{D}^{\alpha}: \bar{B}^{\alpha} \rightarrow R^{n}$ constructed above may be repeated for an arbitrary smooth manifold. In addition, if two such manifolds $M_{i}^{n}, i=1,2$, are close, then their partitions and maps $B_{i}^{\alpha}$ and $D_{i}^{\alpha}$ also can be chosen close to each other. This naturally provides the proof of the fact that the volume is a continuous function on the subset of all manifolds with uniformly bounded from below injectivity radius, bounded diameter and absolute value of the sectional curvature. Below we prove that the volume of a manifold $M^{n}$ is sufficiently close to the volume of $S^{n}$, assuming only that $K_{o}^{\min } \geq 1$ and the Gromov-Hausdorff distance between $M^{n}$ and $S^{n}$ is small.

Volume comparison. Now note that on a subset $B^{\alpha}$ in $M^{n}$ sufficiently close to $\bar{B}^{\alpha}$ we could define the map $D^{\alpha}: B^{\alpha} \rightarrow R^{n}$ similarly to $\bar{D}^{\alpha}: \bar{B}^{\alpha} \rightarrow R^{n}$ in the following way. Take points $p_{i}^{\alpha}$ in $M^{n}$ which are $\epsilon$-close to $\bar{p}_{i}^{\alpha}$ in $S^{n}$ and denote as before by $D^{\alpha}$ the map sending a point $z$ of $B^{\alpha}$ to $\left\{d_{1}^{\alpha}(z), \ldots, d_{n}^{\alpha}(z)\right\}$ where $d_{i}^{\alpha}(z)=$ $\operatorname{dist}_{M^{n}}\left(z, p_{i}^{\alpha}\right)^{5}$. As above, the functions $d_{i}^{\alpha}(z)$ are almost everywhere differentiable and such that their gradient fields $X_{i}$ are unit fields almost everywhere. Therefore, the Jacobian of the map $D^{\alpha}$ is almost everywhere not bigger than 1, i.e., this map is volume contracting, and we arrive at the following statement.

Lemma 13. $\operatorname{vol}\left(B^{\alpha}\right) \geq \operatorname{vol}\left(D^{\alpha}\left(B^{\alpha}\right)\right)$.

Proof. For almost all points $z$ in $B^{\alpha}$ a minimal geodesic $\gamma_{z, i}$ in $M^{n}$ connecting $z$ and $p_{i}^{\alpha}$ is unique. The distance function $d_{i}^{\alpha}$ is differentiable at the point $z$, and for its derivative in a direction $X$ we have by the first variation formula $X d_{i}^{\alpha}=\left(X, X_{i}\right)$, where we denote by $X_{i}$ the direction of $\gamma_{z, i}$ at the point $z$; see (6.5) of [MM]. Hence, taking some orthonormal base $\left\{E_{i}, i=1, \ldots, n\right\}$ at the point $z$, we see that the Jacobian of the map $D^{\alpha}$ has a matrix

$$
\left(d D^{\alpha}\right)_{i j}=\left(E_{k}, X_{i}\right) .
$$

Because all vectors $X_{i}$ have unit length, the determinant of $d D^{\alpha}$ is not bigger than 1. Lemma 13 is proved.

\footnotetext{
${ }^{5}$ Another choice which is interesting to consider is $d_{i}^{\alpha}(z)=\operatorname{dist}_{Z}\left(z, \bar{p}_{i}^{\alpha}\right)$.
} 
If the set $B^{\alpha}$ is close to $\bar{B}^{\alpha}$, its image $D^{\alpha}\left(B^{\alpha}\right)$ is also close to the image $\bar{D}^{\alpha}\left(\bar{B}^{\alpha}\right)$. Generally, this does not guarantee that the volume $D^{\alpha}\left(B^{\alpha}\right)$ is close to the volume of $\bar{D}^{\alpha}\left(\bar{B}^{\alpha}\right)$. But this occurs if we define sets $B^{\alpha}$ in a such way that their images would cover substantial parts of corresponding $\bar{B}^{\alpha}$. In order to do this we use the above constructed map $V^{*}: S^{n} \rightarrow M^{n}$ almost inverse to the map $V: M^{n} \rightarrow S^{n}$.

Definition 2. $B^{\alpha}=V^{*}\left(\left(\bar{B}^{\alpha}\right)_{2 \Lambda_{9}(\epsilon)}\right)$.

First note that due to Lemma 10 sets $B^{\alpha}$ do not intersect. Therefore, the following is true.

\section{Lemma 14.}

$$
\operatorname{vol}\left(M^{n}\right) \geq \sum_{\alpha} \operatorname{vol}\left(B^{\alpha}\right)
$$

From Lemma 10 and the definitions of $\bar{D}^{\alpha}$ and $D^{\alpha}$ we also immediately deduce the following.

Lemma 15. $\operatorname{dist}\left(\bar{D}^{\alpha}(\tilde{x}), D^{\alpha} \circ V^{*}(\tilde{x})\right)<\Lambda_{15}(\epsilon)$, for some $\Lambda_{15}(\epsilon)$ of order $\sigma$ as $\epsilon \rightarrow 0$.

The next lemma shows by simple topological reasoning that, because the map $V^{*}$ is continuous, the images $D^{\alpha}\left(B^{\alpha}\right)$ cover substantial parts of the corresponding images $\bar{D}^{\alpha}\left(\bar{B}^{\alpha}\right)$.

Lemma 16. $D^{\alpha}\left(B^{\alpha}\right) \supset\left(\bar{D}^{\alpha}\left(\left(\bar{B}^{\alpha}\right)_{2 \Lambda_{10}(\epsilon)}\right)\right)_{\Lambda_{15}(\epsilon)}$.

Proof. For every $\alpha$ the images $\bar{D}^{\alpha}\left(\left(\bar{B}^{\alpha}\right)_{2 \Lambda_{10}(\epsilon)}\right)$ and $D^{\alpha}\left(B^{\alpha}\right)$ are images of a topological ball $\left(\bar{B}^{\alpha}\right)_{2 \Lambda_{10}(\epsilon)}$ under, correspondingly, a homeomorphism $H_{0}=\bar{D}^{\alpha}$ and a continuous map $H_{1}=D^{\alpha} \circ V^{*}$. By Lemma 15 these maps are close,

$$
\operatorname{dist}\left(H_{0}(\bar{x}), H_{1}(\bar{x})\right) \leq \Lambda_{15}(\epsilon) .
$$

If $H_{s}(\bar{x})$ denotes the point of an interval connecting $H_{0}(\bar{x})$ and $H_{1}(\bar{x})$ in $R^{n}$ which divides this interval in a ratio $s:(1-s)$, then $H_{s}: \bar{B}^{\alpha} \rightarrow R^{n}$ will be a homotopy between $H_{0}$ and $H_{1}$. From (1.31) it follows that the image of the boundary $\partial \bar{B}^{\alpha}$ of $\bar{B}^{\alpha}$ under this homotopy $H_{s}$ does not intersect with the set $\left(\bar{D}^{\alpha}\left(\left(\bar{B}^{\alpha}\right)_{2 \Lambda_{10}(\epsilon)}\right)\right)_{\Lambda_{15}(\epsilon)}$ of all interior points of $H_{0}\left(\left(\bar{B}^{\alpha}\right)_{2 \Lambda_{10}(\epsilon)}\right)=\bar{D}^{\alpha}\left(\left(\bar{B}^{\alpha}\right)_{2 \Lambda_{10}(\epsilon)}\right)$ having distance not less than $\Lambda_{15}(\epsilon)$ to its boundary, which obviously implies that every point from $\left(\bar{D}^{\alpha}\left(\left(\bar{B}^{\alpha}\right)_{2 \Lambda_{10}(\epsilon)}\right)\right)_{\Lambda_{15}(\epsilon)}$ belongs also to $H_{1}\left(\left(\bar{B}^{\alpha}\right)_{2 \Lambda_{10}(\epsilon)}\right)=D^{\alpha} \circ V^{*}\left(B^{\alpha}\right)$. Lemma 16 is proved.

Now to complete the proof of Theorem $\mathrm{E}$ it is sufficient to note that because the constants $\Lambda_{10}(\epsilon)$ and $\Lambda_{15}(\epsilon)$ are of order $\sigma$ when $\epsilon \rightarrow 0$, i.e., because of $\sigma<<\xi$ they are smaller than the size $\xi$ of $\bar{B}^{\alpha}$ due to the volume estimates of Lemmas 12 and 13 we see that the sum of volumes of $\left(\bar{D}^{\alpha}\left(\left(\bar{B}_{2 \Lambda_{10}(\epsilon)}^{\alpha}\right)\right)_{\Lambda_{15}(\epsilon)}\right)$ tends to the sum of volumes of $\bar{B}^{\alpha}$ when $\epsilon \rightarrow 0$, implying that

$$
\operatorname{vol}\left(M^{n}\right) \geq \operatorname{vol}\left(S^{n}\right)-C(\epsilon)
$$

for some $C(\epsilon) \rightarrow 0$ as $\epsilon \rightarrow 0$. Together with (1.2) this yields the claim of Theorem E. Theorem $\mathrm{E}$ is proved. 


\section{Proof of Theorem $\mathrm{F}$}

As we already mentioned, the Theorem F follows from MS (see Lemma 3.3) with one minor modification. For the reader's convenience, we give here a complete proof, repeating the arguments from [MS].

Let $U \subset T_{o} M^{n}$ be a starshaped open disk domain whose boundary is the tangential cut locus to $o$. For $\tilde{U}=\exp _{o}(U)$ we see that $M^{n} \backslash \tilde{U}=\operatorname{cutlocus}(o)$ has no interior points, and

$$
\operatorname{vol}(\tilde{U})=\operatorname{vol}\left(M^{n}\right)
$$

Define a map $W: S^{n} \backslash\{\bar{o}\} \rightarrow M^{n}$ by $W(\bar{x})=\exp _{o} \circ I \circ \exp _{\bar{o}}^{-1}(\bar{x})$, where $I:$ $T_{\bar{o}} S^{n} \rightarrow T_{o} M^{n}$ is some isometry. Setting $\bar{U}=\exp _{\bar{o}} \circ I^{-1}(U)$, we see that $W$ is a diffeomorphism between $\bar{U}$ and $\tilde{U}$. It is easy to see that Proposition 1 is equivalent to saying that $W$ does not increase distances on $\bar{U}$. Therefore, for an arbitrary subdomain $\bar{D}$ of $\bar{U}$ we have

$$
\operatorname{vol}(W(\bar{D})) \leq \operatorname{vol}(\bar{D})
$$

Because $W$ is a diffeomorphism between $\bar{U}$ and $\tilde{U}$, we have $W^{-1}(\tilde{U} \backslash W(\bar{D}))=$ $\bar{U} \backslash(\bar{D})$. Hence, due to $(2.2)$,

$$
\operatorname{vol}(\tilde{U} \backslash W(\bar{D})) \leq \operatorname{vol}(\bar{U} \backslash \bar{D}) .
$$

Combining (2.1)-(2.3) with the condition $\operatorname{vol}\left(S^{n}\right)-\epsilon \leq \operatorname{vol}\left(M^{n}\right) \leq \operatorname{vol}\left(S^{n}\right)$ of Theorem F, we conclude that for an arbitrary domain $\bar{D}$ in $S^{n}$

$$
\operatorname{vol}(\bar{D})-\epsilon \leq \operatorname{vol}(W(\bar{D})) \leq \operatorname{vol}(\bar{D}) .
$$

Denote by $\omega(\delta)$ the volume of a ball of radius $\delta$ in $S^{n}$. The last inequality implies the following lemma.

Lemma 2.1 (see Lemma 3.2, $\underline{\mathrm{MS}})$. Let $\operatorname{vol}\left(M^{n}\right) \geq \operatorname{vol}\left(S^{n}\right)-\epsilon$. Then $\bar{U}$ is $\delta(\epsilon)$ dense in $S^{n}$ for $\delta(\epsilon)$ such that $\omega(\delta(\epsilon))=\epsilon$.

Proof. If the ball $B(\bar{x}, \delta)$ has no intersection with $\bar{U}$, then

$$
\operatorname{vol}(\bar{U}) \leq \operatorname{vol}\left(S^{n} \backslash B(\bar{x}, \delta)\right)=\operatorname{vol}\left(S^{n}\right)-\omega(\delta) .
$$

Due to $\operatorname{vol}\left(M^{n}\right) \geq \operatorname{vol}\left(S^{n}\right)-\epsilon$ and $(2.1),(2.2)$ we have $\omega(\delta) \leq \epsilon$, and the lemma follows.

Recall that a map $f: X \rightarrow Y$ (not necessary continuous) is said to be an $\epsilon$-approximation if the image $f(X)$ is $\epsilon$-dense in $Y$ and for any $x, y \in X$ we have

$$
|\operatorname{dist}(f(x), f(y))-\operatorname{dist}(x, y)|<\epsilon .
$$

In an equivalent way the distance $d_{G H}(X, Y)$ can be defined as the infimum of the values of $\epsilon>0$ such that there exist $\epsilon$-approximations $f: X \rightarrow Y$ and $g: Y \rightarrow X$. Because in our case both $M^{n}$ and $S^{n}$ have diameters uniformly bounded from above, $\operatorname{diam}\left(M^{n}\right), \operatorname{diam}\left(S^{n}\right) \leq \pi$; we see that the Gromov-Hausdorff convergence $d_{G H}\left(M^{n}, S^{n}\right)<\tilde{C}(\epsilon)$ for some $\tilde{C}(\epsilon) \rightarrow 0$ as $\epsilon \rightarrow 0$ is equivalent to the existence of maps $f: M^{n} \rightarrow S^{n}$ and $g: S^{n} \rightarrow M^{n}$ such that for some $C(\epsilon) \rightarrow 0$ as $\epsilon \rightarrow 0$ the 
following is true:

$$
\begin{aligned}
& 1-C(\epsilon)<\frac{\operatorname{dist}(f(x), f(y))}{\operatorname{dist}(x, y)}<1+C(\epsilon), \\
& 1-C(\epsilon)<\frac{\operatorname{dist}(g(\bar{x}), g(\bar{y}))}{\operatorname{dist}(\bar{x}, \bar{y})}<1+C(\epsilon),
\end{aligned}
$$

for arbitrary $x, y \in M^{n}$ and $\bar{x}, \bar{y} \in S^{n} \operatorname{such}$ that $\operatorname{dist}(x, y), \operatorname{dist}(\bar{x}, \bar{y})>C(\epsilon)$. We call such maps also $C(\epsilon)$-approximations. First we verify that the map $W: S^{n} \rightarrow M^{n}$ satisfies the last property.

Lemma 2.2 (see Lemma 3.3 of $[\mathrm{MS}]$ ). For two points $\bar{x}$ and $\bar{y}$ from $\bar{U}$ such that $\operatorname{dist}(\bar{x}, \bar{y})>\delta^{1 / 2}(4 \epsilon)$,

$$
\frac{\operatorname{dist}(W(\bar{x}), W(\bar{y}))}{\operatorname{dist}(\bar{x}, \bar{y})}>1-\delta^{1 / 2}(4 \epsilon) .
$$

Proof. For $\bar{x}$ and $\bar{y}$ in $S^{n}$ set $2 \bar{r}=\operatorname{dist}(\bar{x}, \bar{y})$ for $\bar{r}>\sigma$. The balls $\bar{B}_{1}=B(\bar{x}, \bar{r})$ and $\bar{B}_{2}=B(\bar{y}, \bar{r})$ are disjoint. Setting

$$
\bar{D}=\left\{\bar{B}_{1} \cup \bar{B}_{2}\right\} \cap \bar{U},
$$

we obtain

$$
\operatorname{vol}(\bar{D}) \geq \operatorname{vol}\left(\bar{B}_{1}\right)+\operatorname{vol}\left(\bar{B}_{2}\right)-\operatorname{vol}\left(S^{n} \backslash \bar{U}\right) .
$$

Because $W$ is volume nonincreasing, we have $\operatorname{vol}(\bar{U}) \geq \operatorname{vol}(\tilde{U})=\operatorname{vol}\left(M^{n}\right) \geq$ $\operatorname{vol}\left(S^{n}\right)-\epsilon$, or

$$
\operatorname{vol}(\bar{D}) \geq \operatorname{vol}\left(\bar{B}_{1}\right)+\operatorname{vol}\left(\bar{B}_{2}\right)-\epsilon=2 \omega(\bar{r})-\epsilon,
$$

and (2.4) implies

$$
\operatorname{vol}(W(D)) \geq 2 \omega(\bar{r})-2 \epsilon .
$$

Denote $x=W(\bar{x}), y=W(\bar{y})$, and let $\bar{z}$ be the midpoint of some minimal geodesic connecting $x$ and $y$. Let $r=\operatorname{dist}(x, y)$. Because $W$ does not increase distances we see that $W(\bar{D}) \subset D$, where

$$
D=B(x, \bar{r}) \cup B(y, \bar{r}) .
$$

Because $r \leq \bar{r}$ an intersection of $B(x, \bar{r})$ and $B(y, \bar{r})$ contains a ball $B=B(z, \bar{r}-r)$. Without loss of generality we can assume that $z \in \tilde{U}$. (If $z \notin \tilde{U}$, i.e., $z \in$ cutlocus $(o)$, we easily find $z^{\prime} \in \tilde{U}$ sufficiently close to $z$ and such that $B\left(z^{\prime}, r^{\prime}\right)$ is in $D$ for $r^{\prime}$ arbitrarily close to $\bar{r}-r$.) Because $W$ is distance-contracting, the preimage $W^{-1}(B(z, \bar{r}-r) \cap \tilde{U})$ contains $B(\bar{z}, \bar{r}-r) \cap \bar{U}$, where $\bar{z}=W^{-1}(z)$. Because as above in (2.5) we have $\operatorname{vol}(B(\bar{z}, \bar{r}-r) \cap \bar{U}) \geq \operatorname{vol}(B(\bar{z}, \bar{r}-r))-\epsilon$, from (2.4) we obtain

$$
\operatorname{vol}(B) \geq \omega(\bar{r}-r)-2 \epsilon
$$

and

$$
\operatorname{vol}(D) \leq 2 \omega(r)-\omega(\bar{r}-r)+2 \epsilon .
$$

This inequality together with (2.6) leads to

$$
0 \leq \omega(\bar{r})-\omega(r)+\omega(\bar{r}-r) \leq 4 \epsilon,
$$

from which we easily deduce

$$
0 \leq \bar{r}-r \leq \omega^{-1}(4 \epsilon)
$$


Therefore,

$$
\frac{\operatorname{dist}(W(\bar{x}), W(\bar{y}))}{\operatorname{dist}(\bar{x}, \bar{y})}=\frac{r}{\bar{r}}>1-\frac{\omega^{-1}(4 \epsilon)}{\bar{r}}>1-\frac{\delta^{1 / 2}(4 \epsilon)}{\delta(4 \epsilon)}=1-\delta^{1 / 2}(4 \epsilon) .
$$

Lemma 2.2 is proved.

Because $W$ does not increase distances, the last lemma means that $W$ is $\delta^{1 / 2}(4 \epsilon)$ approximation between $S^{n}$ and $M^{n}$ tending to isometry as $\epsilon \rightarrow 0$. Clearly, the map $\tilde{W}: M^{n} \rightarrow S^{n}$ which equals $W^{-1}$ on $\tilde{U}$ and sending cutlocus $\{o\}$ to $-\bar{o}$ provides an $C(\epsilon)$-approximation between $M^{n}$ and $S^{n}$ for $C(\epsilon)=\delta^{1 / 2}(4 \epsilon)\left(1-\delta^{1 / 2}(4 \epsilon)\right)^{-1}$. Because $C(\epsilon) \rightarrow 0$ as $\epsilon \rightarrow 0$, this yields that $d_{G H}\left(M^{n}, S^{n}\right)<\tilde{C}(\epsilon) \rightarrow 0$ for some $\tilde{C}(\epsilon) \rightarrow 0$ as $\epsilon \rightarrow 0$. Theorem $\mathrm{F}$ is proved.

\section{REFERENCES}

[C] J. Cheeger, Critical points of distance functions and applications to geometry, Lecture Notes in Math. 1504 (1991), 1-38. MR 94a:53075

[Cl1] T. H. Colding, Shape of manifolds with positive Ricci curvature, Invent. Math. 124 (1996), 175-191. MR 96k:53027

[Cl2] T. H. Colding, Large manifolds with positive Ricci curvature, Invent. Math. 124 (1996), 193-214. MR 96k:53028

[Cl3] T. H. Colding, Ricci curvature and volume convergence, Ann. of Math. 145 (1997), 477-501. MR 98d:53050

[GP] K. Grove, P. Petersen, A radius sphere theorem, Invent. Mathematicae 112 (1993), 577 - 583. MR 94e:53034

[GS] K. Grove, K. Shiohama, A generalized sphere theorem, Ann. of Math. 126 (1977), 201211. MR 58:18268

[M1] Y. Machigashira, Manifolds with pinched radial curvature, Proc AMS 118 (1993), 979985. MR 93i:53038

[M2] Y. Machigashira, Complete open manifolds of non-negative radial curvature, Pacific J. Math. 165 (1994), 153-160. MR 95h:53054

[MM] V. Marenich, S. J. X. Mendonça, Manifolds with minimal radial curvature bounded from below and big radius, Indiana Univ. Math. J. 48 (1999). CMP 2000:04

[MS] Y. Machigashira and K. Shiohama, Riemannian manifolds with positive radial curvature, Japan J. Math. 19 (1994), 419-430. MR 95f:53080

[OSY] Y. Otsu, K. Shiohama, T. Yamaguchi, A new version of differentiable sphere theorem, Invent. Math. 98 (1989), 219-228. MR 91i:53049

[Y1] T. Yamaguchi, Lipschitz convergence of manifolds of positive Ricci curvature with large volume, Math. Ann. 284 (1989), 423-436. MR 90c:53144

[Y2] T. Yamaguchi, Collapsing and pinching under a lower curvature bound, Ann. of Math. 133 (1991), 317-357. MR 92b:53067

IMECC - UNICAMP, CAMPINAS, BRAZIL

E-mail address: marenich@ime.unicamp.br 